\title{
Detection of $B$. pseudomallei DNA among febrile patients presenting to tertiary hospitals in north eastern Malaysia
}

\author{
MRMAli $^{1,2}$, NManing $^{3}$, CYYean $^{1,4}$
}

\section{Introduction}

Melioidosis, caused by Burkholderiapseudomallei, is an important infection, endemic to many tropical regions including South East Asia and northern Australia. In Malaysia, it is estimated that $19 \%$ of melioidosis patients died within the first 48 hours of admission, due to septic shock. Early detection plays an essential role in patients prognosis but clinical diagnosis of melioidosis is difficult, as other tropical infections, such as leptospirosis, dengue and scrub typhus also portray similar disease manifestations. A better detection method is therefore urgently needed to allow timely diagnosis and accurate treatment for melioidosis. Our study aimed to evaluate the performance of qPCR for the detection of B. pseudomallei among febrile patients who presented to healthcare facilities in north eastern Malaysia.

\section{Methods}

A total of 108 blood samples from febrile patients were collected between April 2016 and May 2017 from Hospital Sains Malaysia, Hospital Raja Perempuan Zainab II and Kota Bharu Public Health Laboratory, Malaysia. Genomic DNA were extracted and analysed using real time qPCR to detect the presence of $B$. pseudomallei. Subsequent clinical data, including related microbiological investigation results of patients with positive assay were retrieved to confirm the molecular findings.

\section{Results}

Three samples $(2.8 \%, \mathrm{n}=3 / 108)$ were found to have detectable B. pseudomalleiDNA. Parallel laboratory investigations revealed that these 3 patients had positive blood cultures. $B$. pseudomallei were isolated in two samples. Meanwhile, the other patient's blood culture grew multiple organisms; Klebsiella ozanae and K. oxytoca. Upon retrieval of extensive clinical data, the qPCR-positive, culture-negative patient was reported to have liver abscesses and underlying diabetes mellitus type II. This scenario is possible as conventional culture methods have limited sensitivity $(\sim 60 \%)$. Therefore, multiple sampling and radiological investigation are recommended in patients with suspected melioidosis. We also found that two of the melioidosis patients also had simultaneous leptospirosis, based on the concurrent leptospiral investigations.

\section{Discussion and Conclusions}

This study provides preliminary data in favour of utilisation of qPCR as a sensitive screening tool for melioidosis diagnosis in laboratories of tertiary hospitals in Malaysia, and other endemic regions. Moreover, it also highlights the possibilities of misdiagnosis of

\footnotetext{
${ }^{1}$ Department of Medical Microbiology \& Parasitology, School of Medical Sciences, Universiti Sains Malaysia

${ }^{2}$ Secretariat National Institutes of Health, Ministry of Health, Malaysia

${ }^{3}$ Microbiology Unit, Department of Pathology, Hospital Raja Perempuan Zainab II, Kota Bharu, Malaysia

${ }^{4}$ Institute for Molecular Medicine Research (INFORMM), Universiti Sains Malaysia.

Address for correspondence: Mr Mohammad Ridhuan Mohd Ali, Department of Medical Microbiology \&

Parasitology, School of Medical Sciences, Universiti Sains Malaysia, Malaysia

. +60133029600 Email: ridhuanali@gmail.com iDttps://orcid.org/0000-0002-5888-3526
} 
melioidosis/leptospirosis, in the event when only a single laboratory test is available or requested. 